\title{
Design and Implementation of Intelligent Robot Arm Structure Information Algorithm
}

\author{
Wei Chen
}

Huaqiao University, Xiamen, Fujian 361021, China

Keywords: Robot, ARM, control circuit.

\begin{abstract}
In this paper, intelligent robot control circuit based on ARM microprocessor adopts scattered structure to realize link control calculation of robot and increases calculation, procession ability of controller. The link position control method based on parameter self-adjustment PID effectively reduces the tracking deviation of robot control system and operation system and enhances control ability and operation precision of the intelligent robot. The intelligent robot adopts modularization scattered structure to make design, which can make expansion and updating for motion function and capacity, which is easy for system maintenance.
\end{abstract}

\section{Introduction}

With the gradual development and innovation in science and technology, development in robot has gradually entered real life of people. Robot has been concerned and obtained application in fields such as military, industry, economy as well as disaster prevention and disaster relief. Intelligent, real-time, flexible, precise and small-scale development of robot in future has become the main topic on study of intelligent robot. It is especially important that control system of intelligent robot regard it as its core part. The calculation ability and control ability of single-chip machine such as traditional AVR etc are relatively worse, complicated structure and large-scale circuit can not meet demand in social life and application. The control system of intelligent robot composed of ARM embedded microprocessor with stronger calculation ability and control ability and Linux operation system with stronger real-time are the development direction of robot in the future [1].

The operation precision of manufacturing system for robot in China has increased by 3 to 4 times than that of past, and this technology has gained universal application. Compared with the robot operation system designed in the past, robot operation system at present adopts scattered control structure, this can not only increase the running speed and processing ability of system. In addition, with the quick development in embedded system and CAN-BUS technology, the scattered control structure development of robot has gained more and more attention. So it forms that main control computer of the upper level is responsible for the whole system management as well as coordinate exchange and differential compensation calculation of locus, so that it can coordinate and cooperate to complete the task, enhance running speed of intelligent robot operation system. In this chapter, it mainly studies control circuit design of intelligent robot, that means the design is based on micro-controller of ARM processor and motion controller of $\mu \mathrm{C} / \mathrm{OS}$-II real-time operation system, it adopts obscure PID parameter to further enhance position procession and control capacity of robot in the design process, meet the multiple calculation and procession ability of intelligent robot in node position control.

\section{System design of intelligent robot}

\subsection{System description of intelligent robot.}

The system structure of intelligent robot studied by this paper is indicated by the following Fig. 1, 5 axis frames construct the complete body of robot, high-precision servo table, control system of robot and upper monitor. The robot like this can mainly complete operation in mechanical fabrication 
implementing high-quality procession for machined parts of different shape and dimension. The design on robot operation system adopts scattered control structure, it can control the control speed of link as well as static error through motor current, speed and position of link control system, and dynamic deviation has gained much improvement [1]. The driving device of robot in this chapter is controlled by electric current loop and speed loop through servo motor drive. Motion controller mainly completes rotating position control of motor to realize scattered control. Motion controller also needs to realize cooperation work with other motion controllers and I/O interface of robot controller as well as makes information exchange with main control computer. It will accept control command of main controller and returns to the current control command of link.

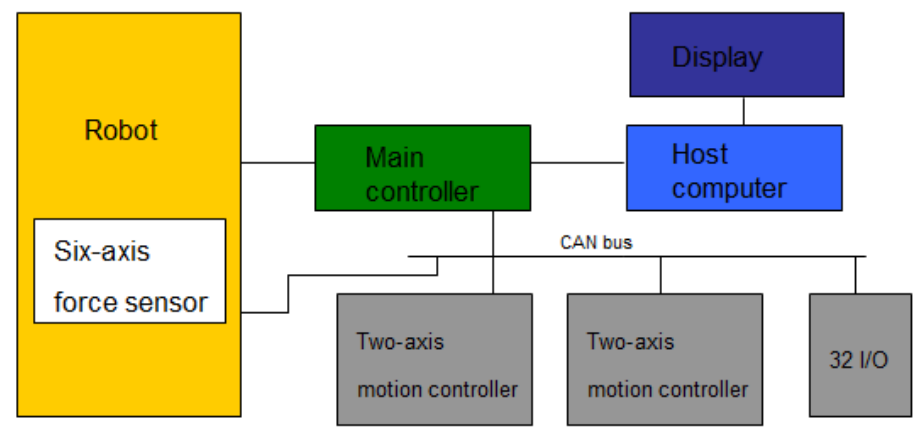

Fig. 1 The overall structure of intelligent robot

\subsection{Hardware design of motion controller.}

2-axis motion controller of intelligent robot uses ARM micro-controller as the core, it adopts CAN bus to make command communication with main controller. For output component of robot, it adopts modularization design idea, uses impulse output component and D/A output component so that it can use standard of different driver interface. Input of code disc adopts incremental and absolute ways to do it. Fig. 2 is structure of motion controller:

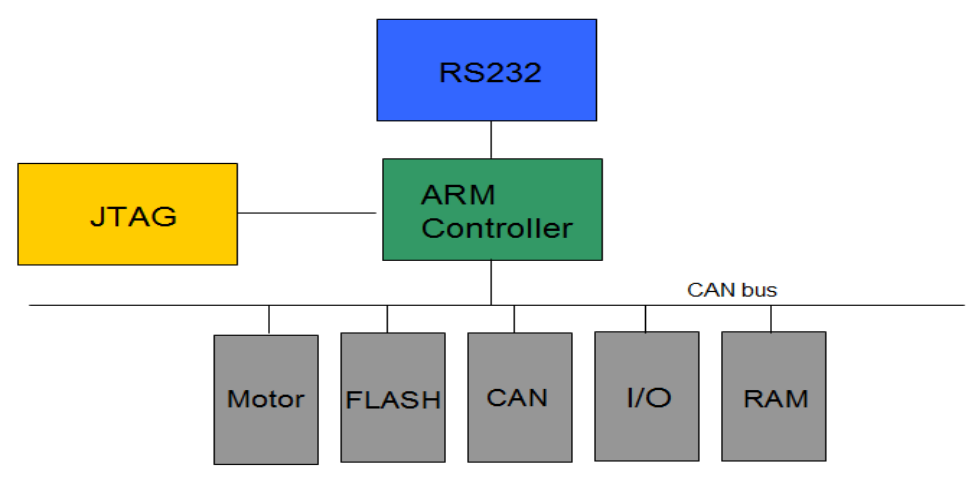

Fig. 2 Structure chart of motion controller

\subsubsection{ARM controller.}

This paper adopts ARM controller of ATMEL Company as the main processor of AT91M40800. It uses high-ability 32 RISC system structure of this processor; it has 16 commands of high-density and low power consumption. Plenty of built-in stack station can make abnormal procession become very quick; it can also make equipment in the real-time control system in the sleep state. ARM also has disk expansion and storage capability in chip such as flash except outer bus interface and flash. It has vectored interrupt controller of 8 grade prior right to cooperate with surrounding equipment data controller so as to enhance real-time [2] of processor to make response to outer interrupt.

\subsubsection{Memorize.}

Memorizer of motion controller includes one piece of 16Mbit flash used as procedure memorizer and one piece of 8MbitSRAM. 


\subsubsection{Change-over circuit of ADC/ DAC.}

Motion controller inputs simulated voltage signal to driver of servo motor as command of motor position, and it can receive the feedback signal transmitted by servo motor by way of simulated voltage signal, so 2-axis motion controller includes 2 ADC and 2 interface transfer circuit.

\subsubsection{Address coding circuit.}

Motion controller can realize address coding in controller through programmable logistics component ALTERA7064.

\subsubsection{Interface circuit of servo motor driver.}

Interface circuit of motor driver includes MC3486differential receiver used as feedback impulse interface of code disc and TLP521 photocoupler used as I/O input and output [3].

\subsubsection{CAN bus control and interface circuit.}

CAN controller uses SLA1000 of Philips and implements bus communication with main controller of robot. It uses data wire D0-D7 of ARM processor as its address or data multiplex line, and it uses data wire of ARM processor as the interface to choose internal interface and data transmission of CAN controller. The ALE, RD, WR signal input and simulated by data wire [4]. Micro-controller and interface of CAN controller can be realized by programmable logic component ALTERA7032. The detailed system logic frame is indicated by Fig. 3.

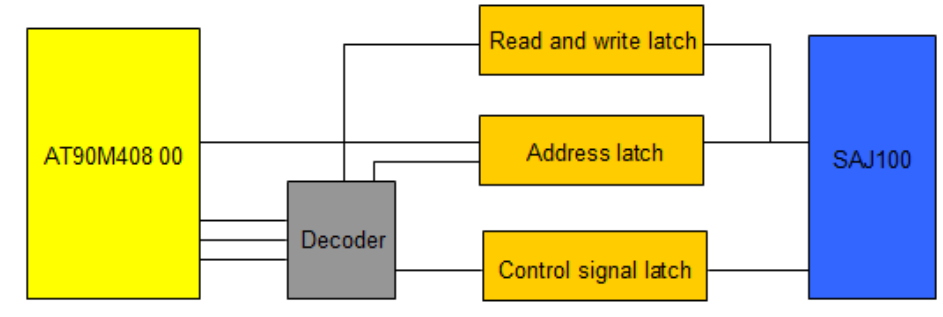

Fig. 3 System logic frame

\subsection{Fuzzy PID parameter control.}

The parameter setting of intelligent robot in motion microprocessor adopts node position control algorithm of fuzzy self-adjustment, it adds to parameter self-adjustment controller on the basis of PID algorithm, it can use fuzzy reasoning according to size, direction of system deviation and change trend etc to make corresponding measures and automatically adjust size of Kp, Ki, Kd of PID, so that it can realize node position of intelligent robot to track deviation reduction. The control system is indicated by the following Fig. 4:

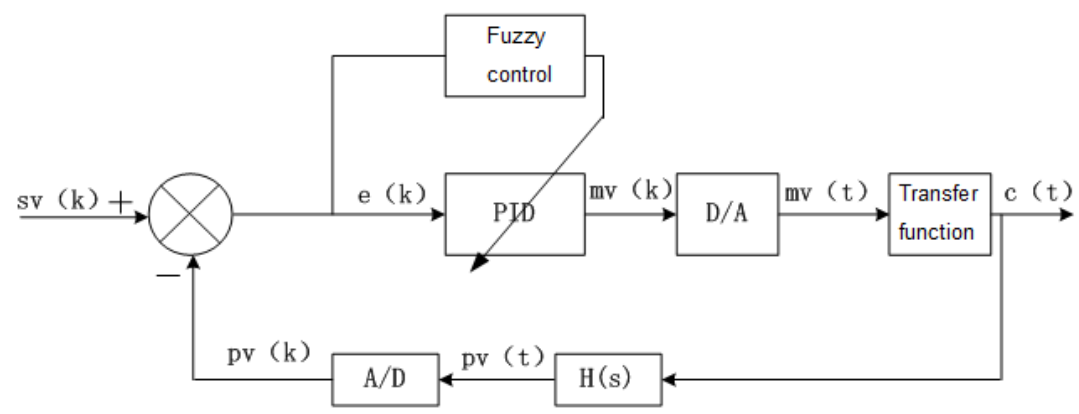

Fig. 4 Control system structure chart

It uses linguistic value of language viable $|\mathrm{e}|$ and $|\mathrm{ec}|$ as large, medium and small and adopts normalization. The affiliated function adopts trigonometric function to balance complete overlap function. It can summarize rule base to adjust PID parameter to adjust PID parameter. 


\section{Experiment test}

Motion controller of intelligent robot uses position control method of self-adjustment PID to make comparative test. The servo motor of intelligent robot adopts servo motor of IFT6070-6ACZ1 without any load. The upper load of robot adopts industrial computer of PC-104 to connect with motion controller by CAN bus, the upper computer use $16 \mathrm{~ms}$ motion peace of control object to send position operation command for robot and controller divides position command of 16ms into 16 , finally it produces one output voltage through close-cycle control calculation in motion controller, and it is used as given amount of controlled motor position cycle, so that it can achieve the aim of controlling link motion of robot. The control coefficient of PID $\mathrm{Kp}=2.0, \mathrm{Kd}=0.36$, the position tracking deviation of robot is indicated by the flowing Fig. 5:

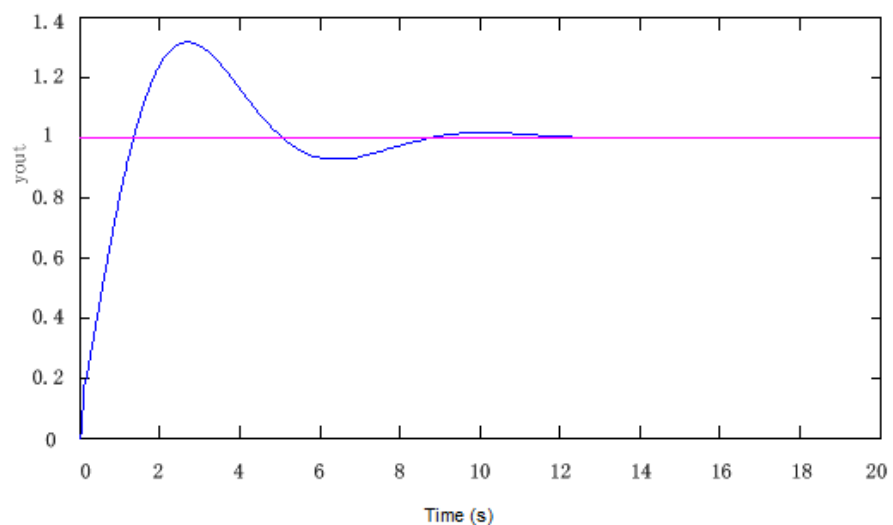

(a) Step response based on common PID

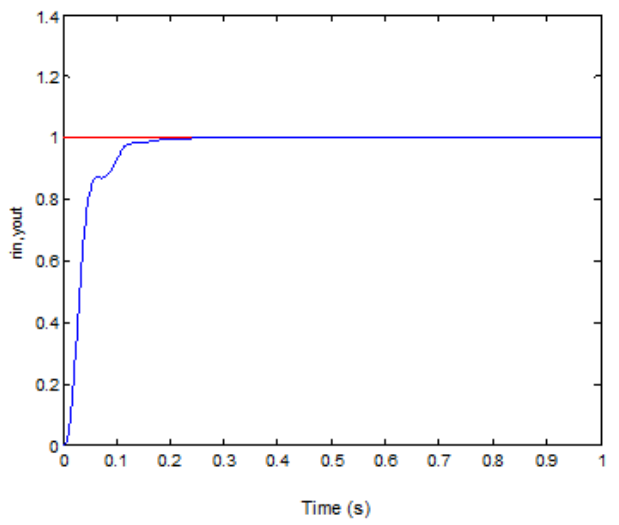

(b) PID step response based on fuzzy control optimization

Fig. 5 Experiment test

Through experiment test, it is difficult to adopt PID method to track link position control and the deviation is very large. While by using fuzzy self-adjustment PID control method, the tracking speed is very quick and tracing deviation is very small. So PID control method of parameter self-adjustment can effectively reduce deviation in position tracking of system and enhance tracking capacity of robot link motion, in addition, the calculation amount is very small and it is easy to realize engineering application.

\section{Conclusion}

According to actual needs, in the machining of actual needs, improve processing efficiency to reduce unnecessary waste of manpower and improve the safety coefficient of mechanical processing. The design of the hardware design of intelligent robot based on ARM control circuit, real-time operating system software using mu C/OS and control joint position of the robot is controlled by using the parameters of the fuzzy PID is introduced and the design. Through the implementation of data show that this control circuit design reduces the operating position of the robot tracking error, improve the ability of robot process control.

\section{References}

[1] Xi'ang Liu, Zhiyu Zhou: Application of Fuzzy Algorithm to a Sonar-Based Obstacle Mobile Robot, Microcomputer Information, (2004) No. 2, p. 31-32. (In Chinese)

[2] Yong Li, Ping Zheng and Li Xiong: Embedded $\mu$ C/OS-II port LPC2104 and communication designing, Microcomputer Information, (2006) No. 02Z, p. 69-71. (In Chinese)

[3] Ze Tian: Embedded system development and Application (Beijing University Press, China 2005).

[4] Ze Chen, Zhenxing Liu and Zongfu Li: ARM embedded technology (Beijing University Press, China 2005). 